\title{
Three-dimensional stability of heated or cooled accelerating boundary layer flows over a compliant boundary
}

$\begin{array}{lll}\text { S. Shateyi* } & \text { P. Sibanda } & \text { S. S. Motsa } \\ & \end{array}$

(Received 14 February 2002, revised 23 June 2002)

\begin{abstract}
In this study we investigate the stability of three-dimensional disturbances imposed on a heated or cooled two-dimensional boundary layer flow with a compliant surface. Such compliant surfaces may delay laminar to turbulent transition and reduce drag and noise levels in fluid flow. We exploit the multi-deck structure of the flow in the limit of large Reynolds numbers to analyse asymptotically the perturbed flow and to derive linear neutral results. A limited parametric study is carried out; the work extends that of Motsa et al. (2002) to three-dimensional disturbances.
\end{abstract}

*Department of Mathematics, University of Zimbabwe, P.O. Box MP 167, Mount Pleasant, Harare, Zimbabwe. mailto:stan@maths.uz.ac.zw

${ }^{\dagger}$ as above. mailto:sibanda@maths.uz.ac.zw

${ }^{\ddagger}$ Department of Mathematics, University of Swaziland, Private Bag 4, Kwaluseni, Swaziland. mailto: sandile@science.uniswa.sz

${ }^{0}$ See http : / / anziamj . austms . org. au/V44/E040 for this article, (c) Austral. Mathematical Soc. 2002. Published July 15, 2002. ISSN 1446-8735 


\section{Contents}

1 Introduction

E56

2 Mathematical Formulation

E58

3 Disturbance Structure

E61

4 Flow Analysis

E61

5 Results

E67

6 Conclusion

E76

A Appendix

E77

References

E78

\section{Introduction}

The stability of boundary layer flow over compliant surfaces has been extensively studied over the past four decades. The huge interest in such flows has been motivated by the potential application of compliant surfaces as a means of delaying laminar to turbulent transition and in reducing drag and noise levels in fluid flow.

The pioneering experimental work on the subject was done by Kramer $[11,12]$ who reported drag reducing capabilities of compliant coatings and conjectured that damping in the compliant coating reduced the growth of Tollmien-Schlichting instability waves in the boundary layer. However, this result was disproved by Benjamin [1] and Landahl [13] who used linear stability theory to study the hydrodynamic stability of the boundary layer flow over a flexible surface. 
They concluded that drag reduction could be achieved in certain types of compliant surfaces by increasing the critical Reynolds number and that damping destabilized the Tollmien-Schlichting waves.

Overwhelming evidence, both experimental (see Grosskreutz [8] and Gaster [7] among others) and theoretical work based on linear stability theory (see Carpenter \& Garrad [2, 3], Sen \& Arora [18], Carpenter \& Morris [4], Yeo [23], Davies \& Carpenter [5] for example), has confirmed that wall compliance can reduce drag forces in fluid motion. As a result, most recent studies have shifted from seeking to establish whether or not compliance reduces drag or delays transition. Attention is now focused on investigating the effect of other factors which may influence the stability of compliant surface flows and in seeking means of optimizing the performance of the compliant surfaces (Dixon et al. [6]) in realistic models. Factors that have been studied so far include nonlinearity (Thomas [21], Rotenberry [17]), boundary layer growth (Yeo [24]), secondary instability (Joslin et al. [10]) and heat transfer (Motsa et al. [15]).

In Motsa et al. [15], the now well known theory of boundary layer flows over heated and cooled surfaces is extended to include surface compliance. The study showed that buoyancy destabilizes the boundary layer flow. The same conclusion was arrived at by Hall \& Morris [9] and Mureithi et al. [16] in the rigid surface case.

In this paper we investigate the effect of wall compliance on the linear stability of three-dimensional disturbances imposed on a twodimensional boundary-layer flow in the presence of buoyancy. The objective of this study is to extend the work of Motsa et al. [15] to three-dimensional disturbances.

Among studies that have been carried out on three-dimensional instabilities are those of Yeo [22, 24] and Carpenter \& Morris [4]. They suggested that compliant walls may be more susceptible to 
three-dimensional instabilities than rigid walls. Yeo [24] showed that the instability of two-dimensional boundary layer flows is characterized by a strong degree of three-dimensionality and that there are no a priori grounds to assume that the most unstable modes will be two-dimensional. In particular, Yeo [24] shows that for sufficiently compliant walls, increasing wall stiffness has the effect of enhancing the dominance of the three-dimensional Tollmien-Schlichting instabilities over the two-dimensional modes.

In this study the main objective is to find out the relative importance of three-dimensional instabilities compared to the two-dimensional modes for a boundary layer flow over a compliant surface with wall heating or cooling.

\section{Mathematical Formulation}

The equations governing three-dimensional disturbances imposed on a heated and or cooled two-dimensional boundary layer flow with a compliant surface in the Boussinesq approximation are:

$$
\begin{aligned}
\frac{\partial u}{\partial t}+u \frac{\partial u}{\partial x}+v \frac{\partial u}{\partial y}+w \frac{\partial u}{\partial z} & =-\frac{\partial p}{\partial x}+\frac{1}{\operatorname{Re}} \nabla^{2} u, \\
\frac{\partial v}{\partial t}+u \frac{\partial v}{\partial x}+v \frac{\partial v}{\partial y}+w \frac{\partial v}{\partial z} & =-\frac{\partial p}{\partial y}-\frac{\alpha g L}{U_{\infty}^{2}}\left(\theta_{\infty}-\theta_{*}\right)+G \theta+\frac{\nabla^{2} v}{\operatorname{Re}}, \\
\frac{\partial w}{\partial t}+u \frac{\partial w}{\partial x}+v \frac{\partial w}{\partial y}+w \frac{\partial w}{\partial z} & =-\frac{\partial p}{\partial z}+\frac{1}{\operatorname{Re}} \nabla^{2} w, \\
\frac{\partial \theta}{\partial t}+u \frac{\partial \theta}{\partial x}+v \frac{\partial \theta}{\partial y}+w \frac{\partial \theta}{\partial z} & =-\frac{1}{\operatorname{Re} \operatorname{Pr}} \nabla^{2} \theta, \\
\frac{\partial u}{\partial x}+\frac{\partial v}{\partial y}+\frac{\partial w}{\partial z} & =0,
\end{aligned}
$$


where $\nabla^{2}=\partial^{2} / \partial x^{2}+\partial^{2} / \partial y^{2}+\partial^{2} / \partial z^{2}$ is the three-dimensional Laplace operator. The parameter $G$ is a buoyancy parameter defined by $G=\operatorname{Gr} / \operatorname{Re}^{2}$ where $\mathrm{Gr}=\alpha g L^{3}\left(\theta_{*}-\theta_{\infty}\right) / \nu^{2}$ is the Grashof number, Pr is the Prandtl number, $\alpha$ is the coefficient of the volume expansion, $\nu$ is the kinematic viscosity and $g$ is the acceleration due to gravity. The velocity has been nondimensionalised by the freestream velocity $U_{\infty}$, the distance by a typical length scale $L$ (for example, the distance measured from the leading edge of the compliant surface), pressure by $\rho_{*} U_{\infty}^{2}$, time by $L / U_{\infty}$ and temperature by $\theta_{*}-\theta_{\infty}$, where $\theta_{\infty}$ is the free-stream temperature and $\theta_{*}$ is the temperature of the plate. Here $\rho_{*}$ is the density at temperature $\theta_{*}$ and the Reynolds number $\operatorname{Re}=U_{\infty} L / \nu$.

For large Reynolds number Re, we define a small parameter $\epsilon=$ $\operatorname{Re}^{-1 / 12}$, the scaled spatial and temporal variables by $x=\epsilon^{5} X$, $z=\epsilon^{5} Z$ and $t=\epsilon^{4} \tau$ respectively. We consider disturbances to the basic flow that are proportional to

$$
E=\exp \left[i \alpha_{0} X+i \beta_{0} Z-i \omega_{0} \tau\right],
$$

where $\alpha_{0}$ and $\beta_{0}$ are respectively the scaled wave numbers in the streamwise and spanwise directions and $\omega_{0}$ is the frequency of disturbances. In order to work with quantities of $\mathcal{O}(1)$ we set

$\alpha=\epsilon^{-5} \alpha_{0}, \quad \beta=\epsilon^{-5} \beta_{0}, \quad c=\epsilon^{-4} c_{0} \quad$ and $\quad \gamma_{0}=\sqrt{\alpha_{0}^{2}+\beta_{0}^{2}}=\epsilon^{5} \gamma$, where $\gamma_{0}$ is the oblique wave number.

We assume that the compliant wall is modelled as an elastic plate (see Motsa et al. [15]) and that the motion of the compliant wall is isotropic, that is, the motion is restricted to the vertical direction. The vertical displacement is represented by $\eta$. The mechanical fluid pressure $\Delta p$ due to $\eta$ is

$$
\Delta p=\frac{T}{\operatorname{Re}^{2}} \nabla^{* 2} \eta-M \frac{\partial^{2} \eta}{\partial t^{2}}-\frac{d}{\operatorname{Re}} \frac{\partial \eta}{\partial t}-\frac{B}{\operatorname{Re}^{2}} \nabla^{* 4} \eta-\frac{K}{\operatorname{Re}^{2}} \eta .
$$


where $\nabla^{* 2}=\partial^{2} / \partial x^{2}+\partial^{2} / \partial z^{2}$. We have non-dimensionalised equation (2) by the following substitutions: $x=x^{\prime} / L, \eta=\eta^{\prime} / L$, $t=t^{\prime} U_{\infty} / L, \Delta p=\delta p^{\prime} / \rho_{*} U_{\infty}^{2}, \theta=\theta^{\prime} \rho_{*} L / \mu_{*}^{2}, K=K^{\prime} L^{3} \rho_{*} / \mu_{*}^{2}$, $M=\rho_{*} b^{\prime} / \rho_{*} L, d=d^{\prime} L / \mu_{*}$ and $B=B^{\prime} \rho_{*} \mu_{*} L$.

At the compliant wall, the boundary condition for the flow is

$$
u=w=0, \quad v=\frac{\partial \eta}{\partial t} \quad \text { at } y=\eta(x, z, t),
$$

where the first boundary condition is the no slip condition at the wall and the second boundary condition is the usual kinematic boundary condition at a movable interface. We use the isothermal boundary condition at the compliant surface for the temperature

$$
\theta=\theta_{B W} \quad \text { at } y=\eta(x, z, t) .
$$

In the far field we assume that the velocity and the temperature approach their free stream values. In the limit as $\operatorname{Re} \rightarrow \infty$, the basic boundary-layer flow takes the form:

$$
\begin{array}{ll}
u=U_{B}(x, Y, z)+\cdots, & v=\operatorname{Re}^{-1 / 2} V_{B}(x, Y, z)+\cdots, \\
w=W_{B}(x, Y, z)+\cdots, & \theta=\Theta_{B}(x, Y, z)+\cdots, \\
p=P_{B}, &
\end{array}
$$

where $Y=\operatorname{Re}^{1 / 2} y$ is the boundary layer coordinate. For general accelerating boundary layers, the basic velocity profile $U_{B}(x, Y, z)$ and temperature profile $\Theta_{B}(x, Y, z)$ have the following additional properties:

$$
\begin{aligned}
U_{B} & \sim \lambda_{1} Y+\lambda_{2} Y^{2}+\cdots \quad \text { as } Y \rightarrow 0, \\
\Theta_{B} & \sim R_{0}+R_{1} Y+R_{2} Y^{2}+\cdots \quad \text { as } Y \rightarrow 0 .
\end{aligned}
$$

In the far-field $U_{B} \rightarrow U_{e}(x), \Theta_{B} \rightarrow 0$ as $Y \rightarrow \infty$ where $U_{e}(x)$ is the nondimensionalised speed of the streaming external flow. The coefficients $\lambda_{1}=\left.U_{B y}\right|_{y=0}>0$ and $\lambda_{2}=\left.U_{B y y}\right|_{y=0}<0$ are respectively 
the skin friction and curvature of the basic flow profile. The coefficients $R_{0}, R_{1}$ and $R_{2}$ are heat transfer coefficients. From Mureithi et al. [16], it is when $G=\mathcal{O}\left(\epsilon^{-5}\right)$ that the Tollmien-Schlichting eigenrelation is first significantly altered. However, at this stage the five-tiered structure of Smith \& Bodonyi [19] persists. We therefore set $G=\epsilon^{-5} G_{0}$ where $G_{0}$ is of $\mathcal{O}(1)$.

\section{Disturbance Structure}

In this work we adopt the five-zone asymptotic structure of Smith \& Bodonyi [19] to investigate the stability of general accelerating boundary layers along the upper-branch of the neutral stability curve. The five regions (see Figure 1) are: the main part of the boundary-layer, Region $\mathrm{R} 1$ of thickness $\mathcal{O}\left(\mathrm{Re}^{-1 / 2}\right)$; a thinner inviscid adjustment Region $\mathrm{R} 2$, of thickness $\mathcal{O}\left(\mathrm{Re}^{-7 / 12}\right)$; which contains the critical-layer Region R3; the viscous wall layer R4 of thickness $\mathcal{O}\left(\mathrm{Re}^{-2 / 3}\right)$; and finally, the outer potential flow Region R5 of thickness $\mathcal{O}\left(\mathrm{Re}^{-5 / 12}\right)$.

We restrict our attention to linear stability and to this end we introduce infinitesimal disturbances of size $\sigma(\ll 1)$ to the basic flow. Smith \& Bodonyi [19] found that linear stability theory holds for disturbance sizes $\sigma$ less than $\mathcal{O}\left(\operatorname{Re}^{-7 / 36}\right)$. It is only when $\sigma$ rises to and beyond $\mathcal{O}\left(\operatorname{Re}^{-7 / 36}\right)$ that nonlinearity comes into play.

\section{Flow Analysis}

Region R1: This region encompasses most of the boundary-layer and is scaled on the thickness of the boundary layer. The appropri- 


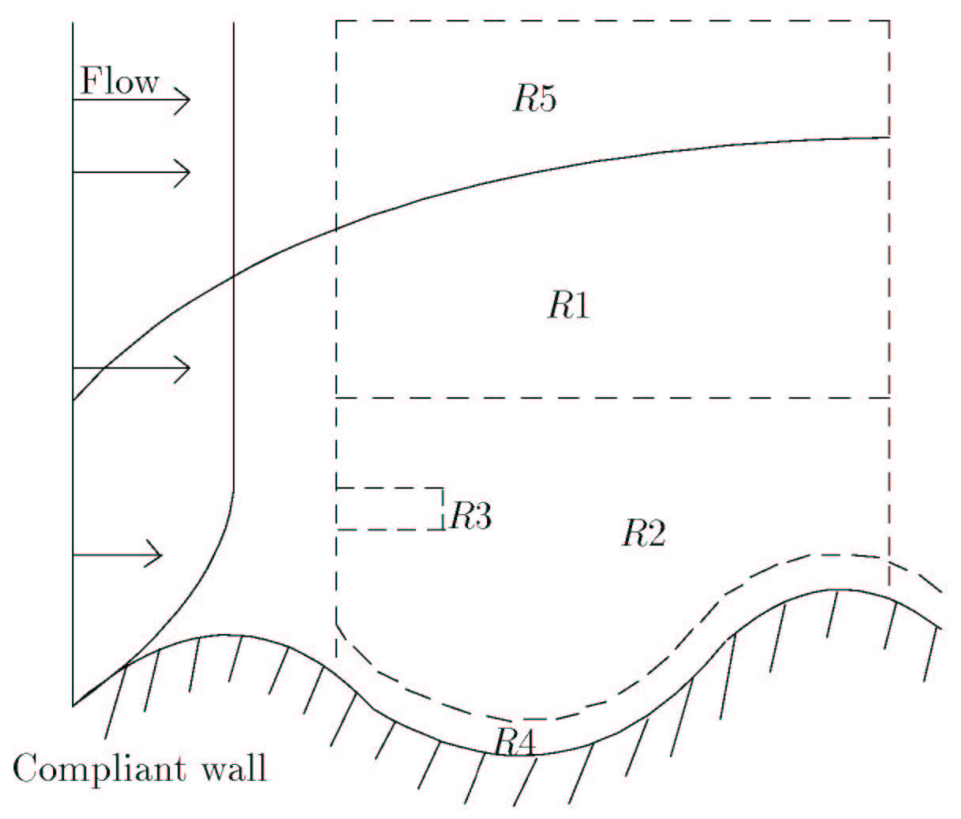

FiguRE 1: Schematic sketch of the multi-deck boundary layer structure. 
ate expansions are:

$$
\left.\begin{array}{l}
u=U_{B}+\sigma\left(u_{0}+\epsilon^{2} u_{1}+\cdots\right), \\
v=\sigma \epsilon\left(v_{0}+\epsilon^{2} v_{1}+\cdots\right), \\
w=\sigma \epsilon^{2}\left(w_{0}+\epsilon^{2} w_{1}+\cdots\right), \\
\theta=\theta_{B}+\sigma\left(\theta_{0}+\epsilon^{2} \theta_{1}+\cdots\right), \\
p=P_{B}+\sigma \epsilon^{2}\left(p_{0}+\epsilon^{2} p_{1}+\cdots\right),
\end{array}\right\}
$$

where the $u_{i}, v_{i}, w_{i}, \theta_{i}$ and $p_{i}$ are functions of the boundary-layer variable $Y$ and of the spatial variable $X$. In order to work with $\mathcal{O}(1)$ terms we define, as earlier, $y=\epsilon^{6} Y$ where $Y=\mathcal{O}(1)$. Substituting equations (6) into the governing equations (1) yields the following leading order solutions for Region R1:

$$
\left.\begin{array}{l}
u_{0}=A_{0} U_{B Y}, \quad v_{0}=-\alpha_{0} A_{0 X} U_{B}, \quad w_{0}=-\frac{\beta_{0} p_{0}}{\alpha_{0} U_{B}} \\
p_{0}=P_{0}+G_{0} A_{0}\left(\theta_{B}-R_{0}\right), \quad \theta_{0}=A_{0} \theta_{B Y} \cdot
\end{array}\right\}
$$

At the next order the solution gives:

$$
\begin{aligned}
v_{1}= & -\alpha_{0} A_{1 X} U_{B}+\alpha_{0} c_{0} A_{0 X}+\frac{\gamma_{0}^{2} U_{B}}{\alpha_{0}} \int_{Y_{0}}^{Y} \frac{P_{0 X}}{U_{B}^{2}} d Y \\
& +\frac{A_{0 X} G_{0} \gamma_{0}^{2} U_{B}}{\alpha_{0}} \int_{Y_{0}}^{Y} \frac{\left(\theta_{B}-R_{0}\right)}{U_{B}^{2}} d Y \\
p_{1}= & P_{1}-\alpha_{0}^{2} A_{0} \int_{0}^{Y} U_{B}^{2} d Y+G_{0} A_{1} \theta_{B} \\
& -\frac{G_{0} \gamma_{0}^{2}}{\alpha_{0}^{2}} \int_{0}^{Y} \theta_{B Y} \int_{Y_{0}}^{Y_{1}} \frac{P_{0}+G_{0} A_{0}\left(\theta_{B}-R_{0}\right)}{U_{B}^{2}} d Y_{1} d Y .
\end{aligned}
$$

where $A_{i}=\bar{A}_{i} e^{i X}+$ c.c., $P_{i}=\bar{P}_{i} e^{i X}+$ c.c. for $i=0,1$ are unknown functions representing the displacement effect and the pressure at the wall respectively and c.c. denotes the complex conjugate. The lower limit of the integrals, $Y_{0}$, is a non-zero constant introduced for convenience, whose value does not affect the eventual results for wave numbers and frequencies. 
Region R2: This is a thin inviscid region of $\mathcal{O}\left(\epsilon^{7}\right)$ that contains the critical layer, Region R3. The critical layer is a region where the phase speed of the disturbance wave is equal to the local flow velocity. We define the boundary-layer coordinate as $y=\epsilon^{7} \bar{Y}$ with $\bar{Y}=\mathcal{O}(1)$ and the expansions become:

$$
\begin{aligned}
& u=\lambda_{1} \epsilon \bar{Y}+\epsilon^{2} \lambda_{2} \bar{Y}^{2}+\sigma\left(u^{(0)}+\epsilon u^{(1)}+\cdots\right) \\
& v=\sigma \epsilon\left(\epsilon v^{(0)}+\epsilon^{2} v^{(1)}+\cdots\right) \\
& w=\sigma\left(w^{(0)}+\epsilon w^{(1)}+\cdots\right) \\
& \theta=R_{0}+\epsilon R_{1} \bar{Y}+\epsilon^{2} R_{2} \bar{Y}^{2}+\sigma\left(\theta^{(0)}+\epsilon \theta^{(1)}+\cdots\right) \\
& p=p_{B}+\sigma\left(\epsilon p^{(0)}+\epsilon^{2} p^{(1)}+\cdots\right) \\
& \eta=\epsilon^{6} \sigma\left(\eta_{0}+\epsilon \eta_{1}+\cdots\right)
\end{aligned}
$$

where $\lambda_{1}=\left.U_{B y}\right|_{y=0}, 2 \lambda_{2}=\left.U_{B y y}\right|_{y=0}$ and $u^{(i)}, v^{(i)}, w^{(i)}, p^{(i)}$ and $\theta^{(i)}$ are functions of $\bar{Y}$ and the spatial variable $X$.

Substituting equation (10) into equations (1) yields the following leading order solutions for Region R2:

$$
\left.\begin{array}{l}
u^{(0)}=\lambda_{1} A_{0}+\frac{\beta_{0}^{2} p^{(0)}}{\alpha_{0}^{2} \lambda_{1} \xi}, \quad v^{(0)}=-\frac{\gamma_{0}^{2} p_{X}^{(0)}}{\lambda_{1} \alpha_{0}}-\alpha_{0} A_{0 X} \lambda_{1} \xi, \\
w^{(0)}=-\frac{\beta_{0} p^{(0)}}{\alpha_{0} \lambda_{1} \xi}, \quad \theta^{(0)}=R_{1}\left(A_{0}+\frac{\gamma_{0}^{2} p^{(0)}}{\alpha_{0}^{2} \lambda_{1}^{2} \xi}\right), \quad p^{(0)}=P^{(0)}
\end{array}\right\}
$$

where $\xi=\left(\bar{Y}-c_{0} / \lambda_{1}\right)$. Using the boundary condition (3) gives

$$
P^{(0)}=\frac{\lambda_{1} \alpha_{0}^{2} c_{0}}{\gamma_{0}^{2}}\left(A_{0}+\eta_{0}\right)
$$

At the next order we obtain:

$$
\begin{aligned}
v^{(1)}= & -\frac{\gamma_{0}^{2} P_{X}^{(1)}}{\alpha_{0} \lambda_{1}}-\alpha_{0} \lambda_{2} A_{0 X}\left(\xi^{2}+\frac{2 c_{0}}{\lambda_{1}} \xi\left\{\ln |\xi|+\phi^{ \pm}\right\}-\frac{c_{0}^{2}}{\lambda_{1}^{2}}\right) \\
& -\frac{2 \lambda_{2} \alpha_{0} c_{0} \eta_{0 X}}{\lambda_{1}}\left(\xi\left\{\ln |\xi|+\phi^{ \pm}\right\}-\frac{c_{0}}{\lambda_{1}}\right)
\end{aligned}
$$




$$
\begin{aligned}
& -\bar{A}_{1 X} \alpha_{0} \xi \lambda_{1}-\frac{\gamma_{0}^{2} G_{0} R_{1} c_{0} A_{0 X}}{\alpha_{0} \lambda_{1}^{2}} \\
& +\frac{\gamma_{0}^{2} G_{0} R_{1}}{\alpha_{0} \lambda_{1}}\left(A_{0 X} \xi\left\{\ln |\xi|+\phi^{ \pm}\right\}\right. \\
& \left.-\frac{c_{0}}{\lambda_{1}}\left(A_{0 X}+\eta_{0 X}\right)\left(\left\{\ln |\xi|+\phi^{ \pm}\right\}+1\right)\right),
\end{aligned}
$$

$$
\begin{aligned}
p^{(1)}= & P^{(1)} \\
& +G_{0} R_{1}\left[A_{0}\left(\xi+\frac{c_{0}}{\lambda_{1}}\right)+\frac{c_{0}}{\lambda_{1}}\left(A_{0}+\eta_{0}\right)\left\{\ln |\xi|+\phi_{p}^{ \pm}\right\}\right]
\end{aligned}
$$

where $P^{(1)}=P^{(1)}(X)$. The solutions in this region possess both logarithmic and algebraic singularities as $\xi \rightarrow 0$. These singularities are smoothed out by the introduction of the critical-layer where $\phi^{ \pm}$ and $\phi_{p}^{ \pm}$are the phase-shift terms introduced to connect the solutions in the normal velocity and pressure respectively on either side of the critical-layer. A reader interested in the different aspects and properties of critical-layers, may, for example, see the review articles by Stewartson [20] and Maslowe [14].

The compliant wall: Equation (2) can be written in the form,

$$
\delta p=p^{\prime}=\bar{T} \epsilon^{5} \nabla^{* 2} \eta-M_{s} \epsilon^{3} \eta_{t t}-\bar{d} \epsilon \eta_{t}-B_{s} \epsilon^{15} \nabla^{* 4} \eta-k_{s} \epsilon^{-5} \eta,
$$

where the constants $\bar{T}, M_{s}, B_{s}, d$ and $k_{s}$ are related to the original parameters by $k_{s}=K \epsilon^{5} / \operatorname{Re}^{2}, B_{s}=B \epsilon^{-15} / \operatorname{Re}^{2}, M_{s}=M \epsilon^{-3}, \bar{T}=$ $T \epsilon^{-5} / \operatorname{Re}^{2}, \bar{d}=d \epsilon / \operatorname{Re}$. This choice of scalings enables the scaled parameters to appear as $\mathcal{O}(1)$ constants in the eigenvalue relation and therefore allows a greater range of compliant properties to be studied. The fluctuating pressure at the wall $p_{*}$ and the vertical displacement $\eta$ are respectively expanded as

$$
p_{*}=\sigma\left(\epsilon \tilde{p}_{0}+\epsilon^{2} \tilde{p}_{1}+\cdots\right), \quad \eta=\sigma \epsilon^{6}\left(\eta_{0}+\epsilon \eta_{1}+\cdots\right),
$$


where we have set $\eta_{i}=\tilde{\eta}_{i} e^{i X}$ for $i=0,1, \ldots$ Using equations (15) and (16) we get

$$
\tilde{p}_{0}=s_{0} \eta_{0}, \quad \tilde{p}_{1}=s_{0} \eta_{1}+d \alpha_{0} c_{0} \eta_{0 X}
$$

where $s_{0}=-\gamma_{0}^{2} \bar{T}+M_{s} \alpha_{0}^{2} c_{0}^{2}-\gamma_{0}^{4} B_{s}-k_{s}$.

Region R4: The solutions found in Region R2 do not satisfy the no slip conditions at the wall. We therefore introduce, as $y \rightarrow$ 0 , a thin viscous layer of thickness $\mathcal{O}\left(\epsilon^{8}\right)$, in which the velocity components adjust to the no-slip condition at the wall. In this region we then set $y=\eta(x, z, t)+\epsilon^{8} \zeta$ where $\zeta$ is an $\mathcal{O}(1)$ coordinate and the flow expansions are:

$$
\left.\begin{array}{l}
u=\lambda_{1} \epsilon^{2} \zeta+\lambda_{2} \epsilon^{4} \zeta^{2}+\cdots+\sigma \tilde{u}_{0}+\cdots, \\
v=\eta_{t}(x, z, t)+\sigma \epsilon^{3} \tilde{v}_{0}+\cdots, \\
w=\sigma \tilde{w}_{0}+\cdots, \\
\theta=R_{0}+R_{1} \epsilon^{2} \zeta+\cdots+\sigma \tilde{\theta}_{0}+\cdots, \\
p=p_{B}+\sigma \tilde{p}_{0}+\cdots, \\
\eta=\sigma \epsilon^{6}\left(\eta_{0}+\epsilon \eta_{1}+\cdots\right),
\end{array}\right\}
$$

where $\tilde{u}_{i}, \tilde{v}_{i}, \tilde{w}_{i}, \tilde{p}_{i}$ and $\tilde{\theta}_{i}$ for $i=0,1,2, \ldots$ are functions of $\zeta$ and $X$. Substituting these expansions into the governing equation (1) and then solving the resulting disturbance differential equations, subject to the boundary conditions at the compliant wall and the matching $($ as $\zeta \rightarrow \infty)$ with the results from Region $\mathrm{R} 2($ as $\bar{Y} \rightarrow 0$ ) yields

$$
\left.\begin{array}{l}
\tilde{v}_{0}=-\frac{i \gamma_{0}^{2} \tilde{p}_{0}}{c_{0} \alpha_{0}}\left(\zeta+\frac{e^{-m \zeta}}{m}-\frac{1}{m}\right)+i \alpha_{0} \lambda_{1} \eta_{0} \zeta, \\
\tilde{\theta}_{0}=-R_{1} \eta_{0} \\
\tilde{p}_{0}=\tilde{P}_{0}
\end{array}\right\}
$$

where $m=\left(\alpha_{0} c_{0}\right)^{1 / 2} e^{-i \pi / 4}$. 
Region R5: This is an outer potential-flow layer in which we define $y=\epsilon^{5} \hat{y}$ where $\hat{y} \sim \mathcal{O}(1)$. The expansions of the perturbations follow from the solutions of Region R1 in the limit $Y \rightarrow \infty$ and are

$$
\left.\begin{array}{l}
u=1+\sigma \epsilon\left(\hat{u}_{0}+\epsilon \hat{u}_{1}+\cdots\right), \\
v=\sigma \epsilon\left(\hat{v}_{0}+\epsilon \hat{v}_{1}+\cdots\right), \\
w=\sigma \epsilon\left(\hat{w}_{0}+\epsilon \hat{w}_{1}+\cdots\right), \\
\theta=\sigma \epsilon\left(\hat{\theta}_{0}+\epsilon \hat{\theta}_{1}+\cdots\right), \\
p=p_{B}+\sigma \epsilon\left(\hat{p}_{0}+\epsilon \hat{p}_{1}+\cdots\right) .
\end{array}\right\}
$$

From these expansions we obtain the following solutions

$$
\hat{u}_{0}=-\hat{P}_{0} e^{-\gamma_{0} \hat{y}}, \quad \hat{v}_{0}=-\frac{i \hat{P}_{0} \gamma_{0} e^{-\gamma_{0} \hat{y}}}{\alpha_{0}}, \quad \hat{p}_{0}=\hat{P}_{0} e^{-\gamma_{0} \hat{y}},
$$

where $\hat{P}_{0}$ is an unknown function which describes the disturbance pressure at the outer extreme of the boundary layer. The important solutions at the next order are:

$$
\begin{aligned}
& \hat{u_{1}}=\left[\hat{P}_{1}-\left(\gamma_{0} \hat{y}-c_{0}\right) \hat{P}_{0}\right] e^{-\gamma_{0} \hat{y}}, \\
& \hat{v}_{1}=-\frac{i \gamma_{0}}{\alpha_{0}}\left[\hat{P}_{1}-\left(\gamma_{0} \hat{y}-\gamma_{0}-c_{0}\right) \hat{P}_{0}\right] e^{-\gamma_{0} \hat{y}}, \\
& \hat{p}_{1}=\left[\hat{P}_{1}-\gamma_{0} \hat{y} \hat{P}_{0}\right] e^{-\gamma_{0} \hat{y}},
\end{aligned}
$$

where $\hat{P}_{1}$ is an unknown function which describes the disturbance pressure at the outer extreme of the boundary layer.

\section{$5 \quad$ Results}

Matching the leading order solutions in the various regions leads to the first dispersion relation

$$
c_{0} \alpha_{0}^{2}=\frac{\left(G_{0} R_{0}+\frac{\alpha_{0}^{2}}{\gamma_{0}}\right)\left(s_{0} \gamma_{0}^{2}-c_{0} \lambda_{1} \alpha_{0}^{2}\right)}{\lambda_{1} s_{0}} .
$$


Matching the second order solutions and restricting ourselves to the $e^{i X}$ components, after some algebra we get:

$$
\begin{aligned}
& i D_{2} A_{0}+i E_{2} G_{0} A_{0}+\frac{\lambda_{1} \alpha_{0}^{2} c_{0}^{2} d \eta_{0}}{s_{0}}-\frac{2 i \alpha_{0} \lambda_{2} c_{0}^{2}}{\lambda_{1}}\left(A_{0}+\eta_{0}\right)\left(\phi^{+}-\phi^{-}\right) \\
& +\frac{2 i \alpha_{0} c_{0} G_{0} R_{1} A_{0}}{\alpha_{0} \lambda_{1}}\left(\phi^{+}-\phi^{-}\right) \\
& -i A_{1}\left(\frac{\gamma_{0}^{2} s_{0}-\lambda_{1} \alpha_{0}^{2} c_{0}}{s_{0} \gamma_{0}}\right)\left(\alpha_{0} U_{B}^{\infty}-\frac{\gamma_{0} G_{0}}{\alpha_{0}}\left(\theta_{B}^{\infty}-\theta_{B}^{0}\right)\right) \\
& +i \alpha_{0} c_{0} \lambda_{1} A_{1} U_{B}^{\infty}-\frac{i \lambda_{1} \gamma_{0}^{2} \tilde{p}_{0}}{\alpha_{0} m c_{0}}=0
\end{aligned}
$$

where the constants $D_{i}$ and $E_{i}$ for $i=0,1 \ldots$, are defined in Appendix A. Since $U_{B}^{\infty}=1, \theta_{B}^{\infty}=0$ and $\theta_{B}^{0}=R_{0}$, the coefficient of $A_{1}$ in the above expression is

$$
i \alpha_{0} c_{0} \lambda_{1}-i \alpha_{0}\left(\frac{\gamma_{0}^{2} s_{0}-\lambda_{1} \alpha_{0}^{2} c_{0}}{s_{0} \gamma_{0}}\right)\left(1+\frac{\gamma_{0} G_{0} R_{0}}{\alpha_{0}^{2}}\right)
$$

which is zero according to the first dispersion relation. The results for linear theory are derived by taking the jump across the critical layer, $\phi$ to be equal to $i \pi$. Taking the real parts of equation (24) then gives the second dispersion relation as:

$$
-\frac{\alpha_{0} \lambda_{1}^{2}}{\sqrt{2} \bar{m}}-\frac{d_{1 r} \lambda_{1}^{2} \alpha_{0}^{4} c_{0}^{3}}{s_{0}^{2} \gamma_{0}^{2}}=\frac{2 \alpha_{0} \lambda_{2} c_{0}^{2} \pi}{\lambda_{1}}-\frac{2 c_{0} G_{0} R_{1} \pi}{\lambda_{1} \alpha_{0}}\left(\frac{s_{0} \gamma_{0}^{2}-c_{0} \lambda_{1} \alpha_{0}^{2}}{s_{0}}\right)
$$

where $d_{1 r}$ is the real part of $d$ and $\bar{m}=\sqrt{\alpha_{0} c_{0}}$.

Equations (23) and (26) are the crucial eigenvalue relations which fix the neutral oblique wavenumber to the neutral wavespeed. If we assume that $\gamma_{0}=\alpha_{0} \cos \psi$ for some angle $\psi$, we get:

$$
c_{0} \lambda_{1} s_{0}=\left(G_{0} R_{0}+\frac{\alpha_{0}}{\cos \psi}\right)\left(s_{0} \cos ^{2} \psi-c_{0} \lambda_{1}\right)
$$




$$
\begin{gathered}
-\frac{\alpha_{0} \lambda_{1}^{2}}{\sqrt{2} \bar{m}}-\frac{d_{1 r} \lambda_{1}^{2} \alpha_{0}^{2} c_{0}^{3}}{s_{0}^{2} \cos ^{2} \psi}=\frac{2 \alpha_{0} \lambda_{2} c_{0}^{2} \pi}{\lambda_{1}} \\
-\frac{2 \alpha_{0} c_{0} G_{0} R_{1} \pi}{\lambda_{1}}\left(\frac{s_{0} \cos ^{2} \psi-c_{0} \lambda_{1}}{s_{0}}\right)
\end{gathered}
$$

where $s_{0}=-\alpha_{0}^{2} \cos ^{2} \psi \bar{T}+M_{s} \alpha_{0}^{2} c_{0}^{2}-\alpha_{0}^{4} \cos ^{4} \psi B_{s}-k_{s}$.

Consider the limiting behaviour of the neutral wavenumber $\alpha_{0}$, and the neutral wave speed $c_{0}$ in the limit $G_{0} \rightarrow \pm \infty$. The physical significance of the limit $G_{0} \rightarrow+\infty\left(G_{0} \rightarrow-\infty\right)$ corresponds to the increase (decrease) in buoyancy force through wall heating (cooling).

Solving the eigenrelations (27) and (28) we get, in the limit $G_{0} \rightarrow$ $+\infty$ with $B_{s}=M_{s}=0, d_{1 r} \geq 0, k_{s} \neq 0$ and $\bar{T} \neq 0$ :

$$
\alpha_{0}=\left(\frac{R_{1} \lambda_{1}}{\lambda_{2}}-R_{0}\right) G_{0} \cos \psi+\cdots, \quad c_{0}=\frac{R_{1}}{\lambda_{2}} G_{0} \cos ^{2} \psi+\cdots .
$$

If we now choose our parameters such that $M_{s}=0, B_{s} \neq 0, d_{1 r} \geq$ $0, k_{s} \neq 0$ and $\bar{T} \neq 0$, then in the limit $G_{0} \rightarrow-\infty$ we get,

$$
\alpha_{0}=-R_{0} G_{0} \cos \psi+\cdots, \quad c_{0}=-\frac{\lambda_{1}^{2}}{\left(8 R_{1}^{2} \pi^{2} \cos ^{5} \psi\right)^{1 / 3}} G_{0}^{-1}+\cdots,
$$

where asymptotic analysis of the eigenrelation gives $\alpha_{0} \sim \mathcal{O}\left(G_{0}\right)$. Since $\alpha_{0}$ increases as $G_{0}$ increases we note that in the limit $G_{0} \rightarrow$ $+\infty$, the neutral wavenumber $\alpha_{0}$ and the neutral wave speed $c_{0}$ increase whereas in the limit $G_{0} \rightarrow-\infty, \alpha_{0}$ increases with $G_{0}$, whereas $c_{0}$ decreases. This suggests that as the buoyancy parameter is further increased, the wavelength of the neutral modes becomes progressively shorter and that a new limit must be reached as $\left|G_{0}\right| \rightarrow \infty$. Mureithi et al. [16] analysed this new distinguished limit and found that as the factor $G$ became large and positive, the flow structure collapsed and become two layered with the disturbances 

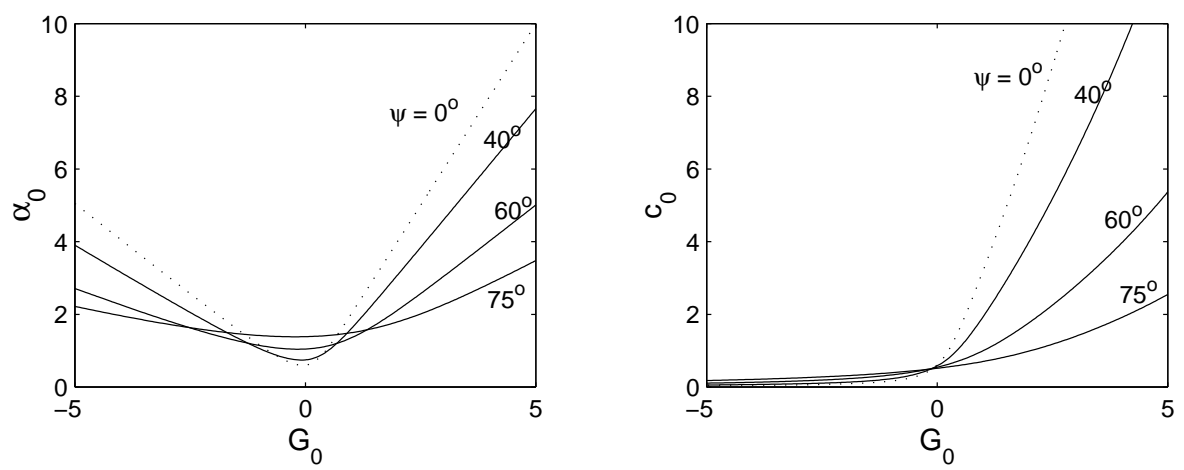

FigURE 2: Linear neutral wavenumber $\alpha_{0}$ against $G_{0}$ with $k_{s}=50$, $d_{1 r}=10, \bar{T}=M_{s}=B_{s}=0$

to the basic flow governed by the classical Taylor-Goldstein equation. Similarly, for $G$ large and negative it was found that the flow structure was two layered with the disturbances to the basic flow governed by the steady Taylor-Goldstein equation in the majority of the boundary layer.

Numerical results are presented in Figures 2-6. Figure 2 shows how the neutral wavenumber $\alpha_{0}$ and neutral wavespeed $c_{0}$ vary with the buoyancy parameter $G_{0}$ for selected values of the wall parameters and for different values of $\psi$. The dotted line shows the corresponding two-dimensional results of Motsa et al. [15]. Note that $\alpha_{0} \rightarrow \infty$ as $\left|G_{0}\right| \rightarrow \infty$ and that $c_{0} \rightarrow \infty$ as $G_{0} \rightarrow \infty$ and $c_{0} \rightarrow 0$ as $G_{0} \rightarrow-\infty$ for all angles $\psi$. The effect of increasing the angle $\psi$ is to reduce the rate at which $\alpha_{0}$ and $c_{0}$ approach infinity as $G_{0}$ becomes large. This means that the rate of growth of the two-dimensional disturbances is larger than that of the oblique modes for marginal heating and cooling. This is consistent with Squire's theorem for flows over rigid surfaces.

Figure 3 represents the results for the variation of the neu- 
tral wavenumber $\alpha_{0}$ with respect to the tension parameter $\bar{T}$ for $k_{s}=100, d_{1 r}=10, G_{0}=0.1, \psi=0,30^{\circ}, 45^{\circ}, 60^{\circ}$ with all other parameters set to zero. See that for fixed values of $k_{s}, d_{1 r}$ and $G_{0}$, small values of the tension parameter produce much larger wavenumbers for two-dimensional disturbances as compared to the three-dimensional modes.

Note also that the effect of increasing $\bar{T}$ is more significant in the three-dimensional modes than in the two-dimensional modes. The same trend was observed when the wavenumber was varied against $B_{s}$ and $k_{s}$. When $\psi$ is further increased (for example when $\psi=60^{\circ}$ ) note that an extra mode is obtained - this mode corresponds to the rigid wall solution.

The above result indicates that an increase in the three-dimensionality of the waves has the same effect as increasing the stiffness (reducing the flexibility) of the compliant surface. This result is in agreement with the results of Yeo [24]. Figure 4 shows the variation of the neutral wavenumber $\alpha_{0}$ against the damping parameter $d_{1 r}$ for fixed values of $k_{s}, G_{0}, d_{1 r}$ and various values of $\psi$ with all other parameters set to zero. Note that the wavenumber is reduced when $\psi$ is increased and that the effect of damping is more pronounced in two-dimensional modes than in three-dimensional modes. Increasing $d_{1 r}$ leads to an effect which is opposite that of increasing the wall parameters $\bar{T}, k_{s}$ and $B_{s}$. This is in line with results from previous studies (see, for example, Carpenter \& Garrad [2]). Figure 5 shows the variation of the wavenumber $\alpha_{0}$ against $\psi$ for different values of the stiffness parameter $k_{s}$ with $G_{0}=0.01$ and $d_{1 r}=100$. The dotted line illustrates the rigid wall case. See that there exists two distinct modes, particularly for small angles. One of the modes (the "lower-branch" of the curve) corresponds to the rigid wall solution and the other mode (the "upper-branch" of the curve) is due to the introduction of wall compliance. Note that as the surface becomes 


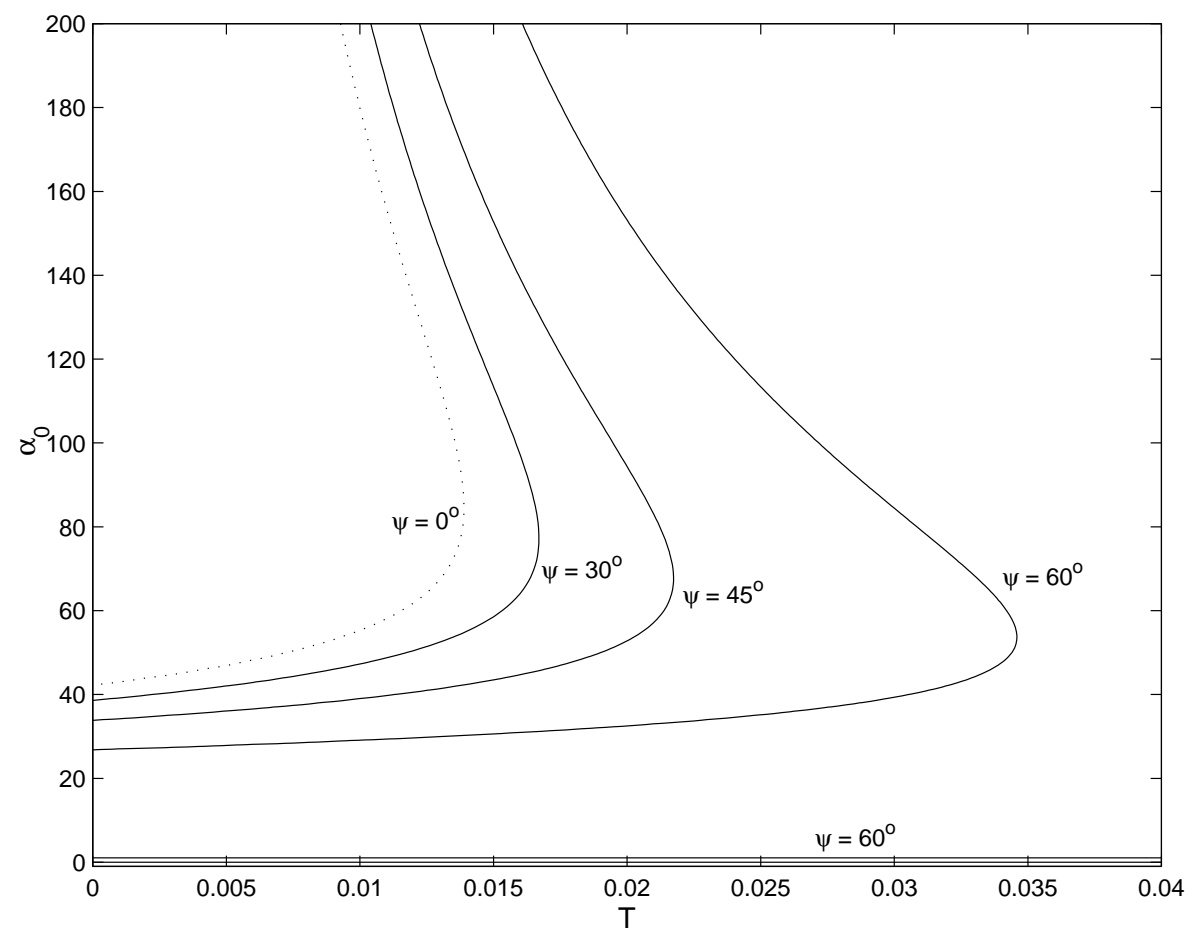

FiguRE 3: Linear neutral wavenumber $\alpha_{0}$ against $\bar{T}$ with $k_{s}=100$, $d_{1 r}=10, G=0.1, B_{s}=M_{s}=0$. 


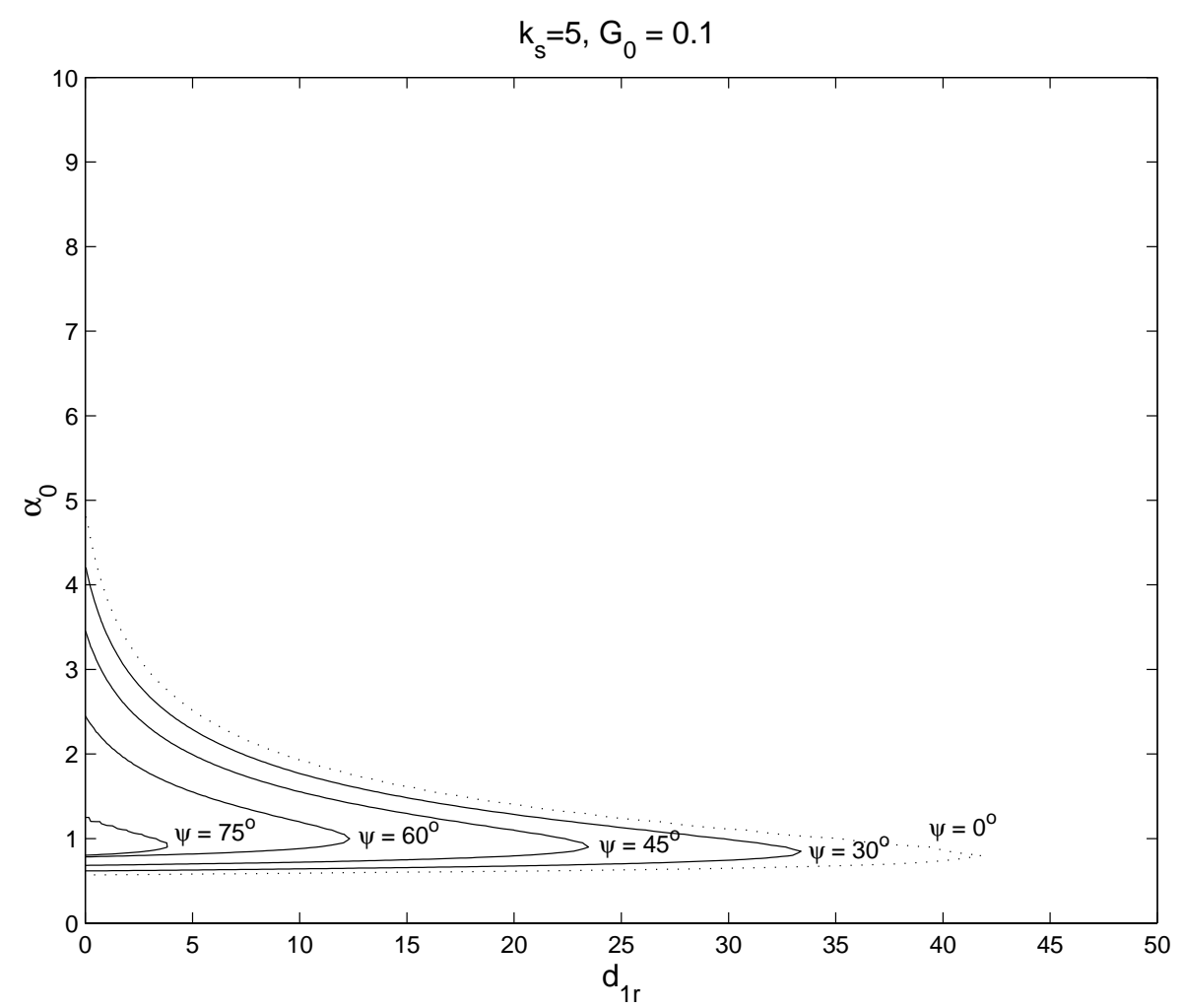

FIGURE 4: Linear neutral wavenumber $\alpha_{0}$ against $d_{1 r}$ with $k_{s}=5$, $\bar{T}=B_{s}=M_{s}=0$. 


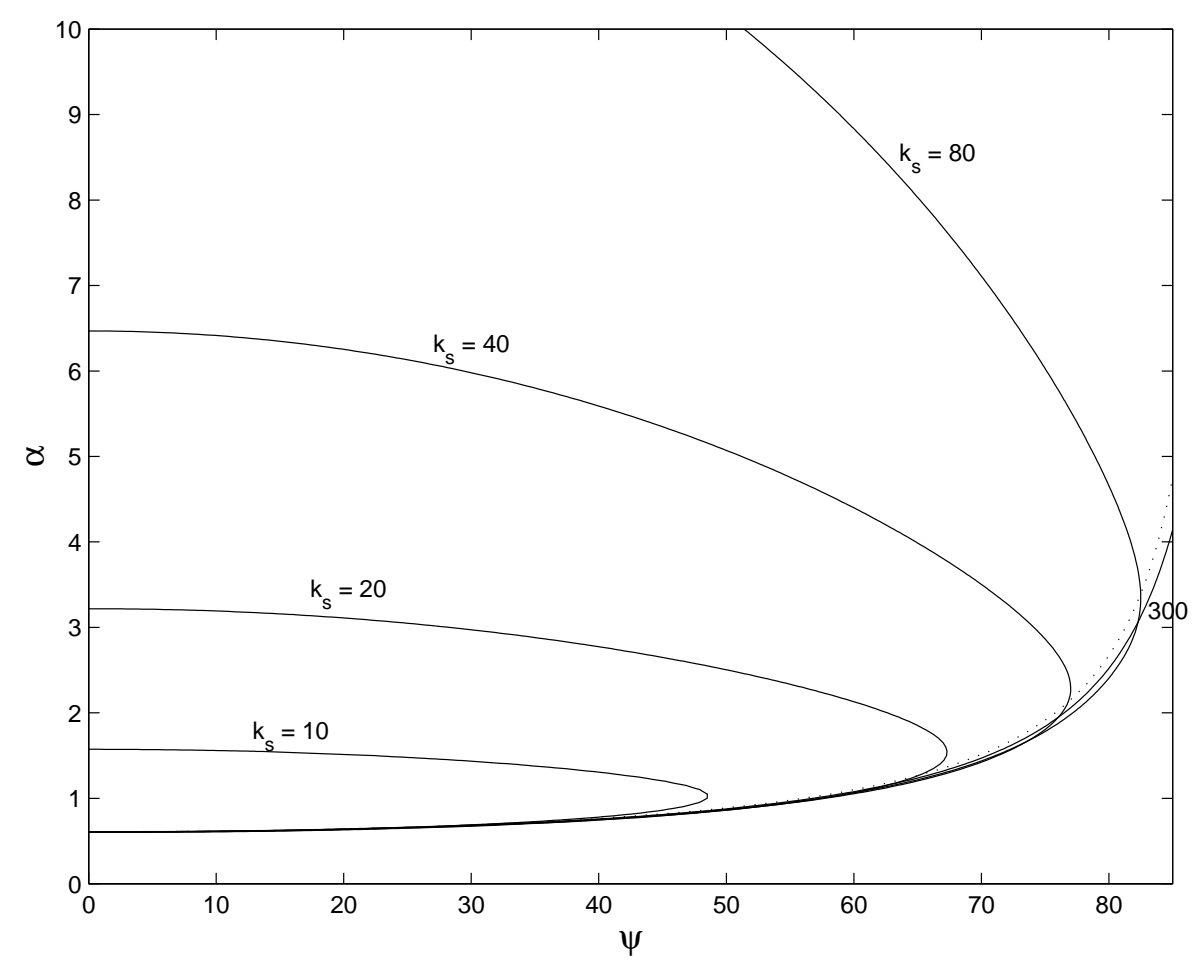

FiguRE 5: Linear neutral wavenumber $\alpha_{0}$ against $\psi$ with $k_{s}=$ $10,20,40,80,300, d_{1 r}=100, G_{0}=0.01, \bar{T}=B_{s}=M_{s}=0$ 


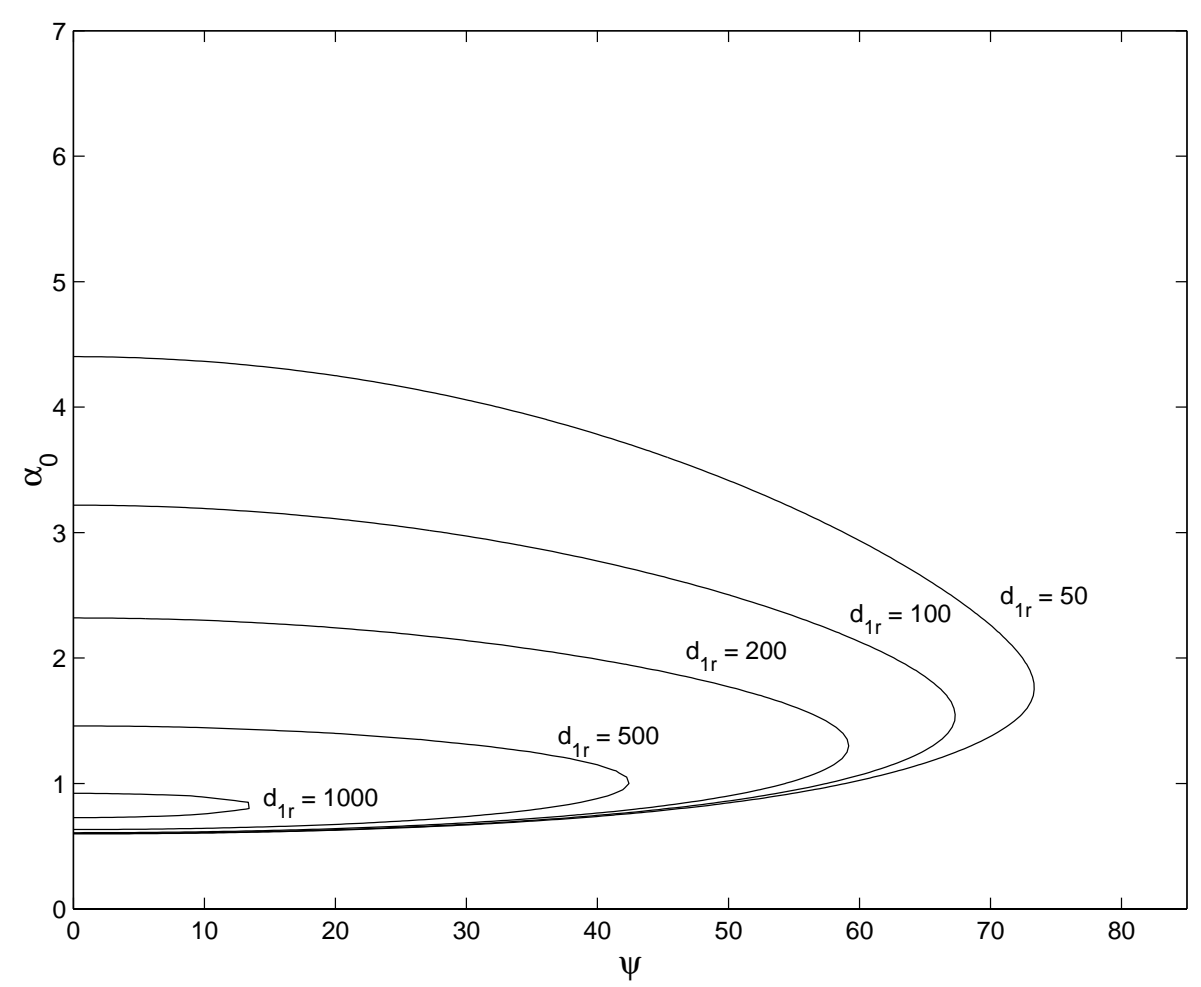

FiguRE 6: Linear neutral wavenumber $\alpha_{0}$ against $\psi$ with $k_{s}=20$, $d_{1 r}=50,100,200,500,1000, G_{0}=0.01, \bar{T}=B_{s}=M_{s}=0$. 
stiff (that is, when $k_{s}$ becomes very large) the compliant wall based mode tends to the rigid wall mode when $\psi$ tends to $\pi / 2$.

Figure 6 represents the results for the variation of the neutral wavenumber $\alpha_{0}$ with respect to the angle $\psi$ for different values of $d_{1 r}$ and $k_{s}=20, G_{0}=0.01$. See in this figure that as $d_{1 r}$ becomes large, the wavenumber is progressively decreased, that is, large damping has a stabilizing influence on the disturbance waves. The rigid wall results are also not recovered when $d_{1 r}$ and $\psi$ become large.

\section{Conclusion}

We have considered the effect of thermal buoyancy on the linear stability of three-dimensional (oblique) disturbance wave modes in two-dimensional boundary layer flows over compliant walls. We have extended the well known theory of boundary layer flows over heated or cooled surfaces to include surface compliance and threedimensionality of the disturbances.

From Figure 2 observe that the rate of growth of two-dimensional disturbances is much larger than that of the oblique disturbances for marginal heating and cooling. This is consistent with Squire's theorem.

When the wavenumber is varied against the tension parameter, spring stiffness and flexural rigidity, the effect of three-dimensional modes is more significant than that of the two-dimensional modes. The opposite effect is observed when the wavenumber was varied against the damping parameter. The dominance of three-dimensional modes when the wall becomes more compliant implies that the prediction of laminar-turbulent transition based solely on twodimensional modes may not give accurate results. This suggests 
that for more realistic prediction, the growth of three-dimensional modes must be considered.

When the wavenumber is varied against the angle $\psi$, the wall becomes more stiff when $\psi$ becomes large. Yeo [24] arrived at the same conclusion.

\section{A Appendix}

The constants as used in the article.

$$
\begin{aligned}
I_{0} & =\int_{0}^{\infty} U_{B}^{2} d Y, \\
I_{1} & =\int_{Y_{0}}^{\infty *} \frac{1}{U_{B}^{2}} d Y, \\
I_{2} & =\int_{Y_{0}}^{0 *} \frac{1}{U_{B}^{2}} d Y, \\
I_{3} & =\int_{Y_{0}}^{0 *} \frac{\left(\theta_{B}-R_{0}\right)}{U_{B}^{2}} d Y, \\
J_{0} & =\int_{0}^{\infty} \theta_{B Y} \int_{Y_{0}}^{Y} \frac{G_{0} R_{0}+\frac{\alpha_{0}^{2}}{\gamma_{0}}+G_{0}\left(\theta_{B}-R_{0}\right)}{U_{B}^{2}} d Y_{1} d Y, \\
J_{1} & =\frac{s_{0} \gamma_{0}^{2}}{\lambda_{1}\left(s_{0} \gamma_{0}^{2}-c_{0} \lambda_{1} \alpha_{0}^{2}\right)}\left\{\ln \left|\frac{c_{0}}{\lambda_{1}}\right|+\left(\phi_{p}^{+}-\phi_{p}^{-}\right)\right\}, \\
J_{2} & =-\left(\frac{R_{1} c_{0}}{\lambda_{1}} J_{1}+\frac{\gamma_{0}^{2}}{\alpha_{0}^{2}} J_{0}\right), \\
J_{3} & =\int_{Y_{0}}^{\infty *} \frac{\theta_{B}-R_{0}}{U_{B}^{2}} d Y, \\
E_{0} & =\frac{\gamma_{0}^{2} \lambda_{1}}{\alpha_{0}}\left(R_{0} I_{2}+I_{3}\right),
\end{aligned}
$$




$$
\begin{aligned}
E_{1}= & -\frac{\gamma_{0}^{2} c_{0} R_{1}}{\alpha_{0} \lambda_{1}^{2}}\left(\ln \left|\frac{c_{0}}{\lambda_{1}}\right|+2+\frac{c_{0} \lambda_{1} \alpha_{0}^{2}}{s_{0} \gamma_{0}^{2}-c_{0} \lambda_{1} \alpha_{0}^{2}}\right), \\
E_{2}= & \lambda_{1} E_{1}-c_{0} E_{0}+\frac{R_{1} C_{0} \gamma_{0}^{2}}{\alpha_{0} \lambda_{1}^{2}} \ln \left|\frac{c_{0}}{\lambda_{1}}\right| \\
& +\frac{s_{0} \gamma_{0}^{2}-\alpha_{0}^{2} \lambda_{1} c_{0}}{s_{0} \alpha_{0}}\left(\frac{\gamma_{0}^{2}}{\alpha_{0}^{2}} J_{0}-\gamma_{0} R_{0} I_{1}\right), \\
D_{0}= & \alpha_{0} \gamma_{0} \lambda_{1} I_{2}-\frac{2 \alpha_{0} c_{0} \lambda_{2}}{\lambda_{1}} \\
D_{1}= & \frac{2 \alpha_{0} c_{0}^{2} \lambda_{2}}{\lambda_{1}^{2}}\left(\ln \left|\frac{c_{0}}{\lambda_{1}}\right|+\left(\frac{s_{0} \lambda_{1} \alpha_{0}^{2}}{s_{0} \gamma_{0}^{2}-c_{0} \lambda_{1} \alpha_{0}^{2}}\right)\left(1+\ln \left|\frac{c_{0}}{\lambda_{1}}\right|\right)\right), \\
D_{2}= & \lambda_{1} D_{1}-c_{0} D_{0}-\left(\frac{\gamma_{0}^{2} s_{0}-\lambda_{1} \alpha_{0}^{2} c_{0}}{s_{0} \alpha_{0}}\right)\left(\alpha_{0} I_{0}-2 c_{0}+\gamma_{0}\right) \\
& -\alpha_{0}^{2} \gamma_{0} c_{0} I_{1} .
\end{aligned}
$$

Acknowledgements: SS and SSM gratefully acknowledge financial support from the Norwegian Council of Universities Committee for Cooperation Research and Development (NUFU).

\section{References}

[1] T. B. Benjamin. Effects of a flexible boundary on hydrodynamic stability. J. Fluid Mech. 9 (1960) 513-532. E56

[2] P.W. Carpenter and A. D. Garrad. The hydrodynamic stability flow over Kramer type compliant surfaces. Part 1. Tollmien-Schlichting instabilities. J. Fluid Mech. 155 (1985) 465-510. E57, E71 
[3] P.W. Carpenter and A. D. Garrad. The hydrodynamic stability flow over Kramer type compliant surfaces. Part 2. Flow-induced surface instabilities. J. Fluid Mech. 170 (1986) 199-232. E57

[4] P. W. Carpenter and P. J. Morris. Effect of anisotropic wall compliance on boundary layer stability and transition. $J$. Fluid Mech. 218 (1990) 171-223. E57

[5] C. Davies and P. W. Carpenter. Instabilities in plane channel flow between compliant walls. J. Fluid Mech. $\mathbf{3 5 2}$ (1997) 205-243. E57

[6] A. E. Dixon, A. D. Lucey and P. W. Carpenter. Optimization of viscoelastic compliant walls for transition delay. AIAA Journal 32 (1994) 256-267. E57

[7] M. Gaster. Is the dolphin a red herring? In H.W Liepmann and R. Narisimha, editors, Proceedings of the IUTAM Conference on Turbulence Management and Relamination, pages 285-304. Bangalore, India, 1987. E57

[8] R. Grosskreutz. An attempt to control boundary-layer turbulence with nonisotropic compliant walls. Univ. Sci. Jour., Dar es Salaam 1 (1975) 67. E57

[9] P. Hall and Morris H. On the instability of boundary layers on heated flat plates. J. Fluid Mech. 245 (1992) 367-400. E57

[10] R. D. Joslin, P. J. Morris and P. W. Carpenter. The role of three-dimensional instabilities in compliant wall boundary layer transition. AIAA Journal 29(10) (1991) 1603-1610. E57

[11] M. O. Kramer. The dolphin's secret. New Scientist 7 (1960) 1118-1120. E56 
[12] M. O. Kramer. Boundary layer stabilization by distributed damping. J. Am. Soc. Naval Maths 58 (1962) 19-50. E56

[13] M. T. Landahl. On the stability of laminar incompressible boundary layer over a flexible surface. J. Fluid Mech. 13 (1962) 609-632. E56

[14] S. Maslowe. Critical layers in shear flows. Ann. Rev. Fluid Mech. 18 (1986) 405-432. E65

[15] S. S. Motsa, J. S. B. Gajjar and P. Sibanda. On the upper-branch stability of boundary-layer flows over compliant surfaces with heating/cooling. Computer Assisted Mechanics and Engineering Sciences 9(2) (2002) 163-181. E57, E59, E70

[16] E. W. Mureithi, J. P. Denier and A. K. Stott. The effect of buoyancy on the upper-branch Tollmien- Schlichting waves. IMA J. Appl. Math. 58 (1997) 19-50. E57, E61, E69

[17] J. M. Rotenberry. Finite-amplitude shear waves in channels with compliant boundaries. Phys. Fluids A 4(2) (1992) 270-276. E57

[18] P. K. Sen and D. S. Arora. On the stability of boundary-layer flow over a flat plate with a compliant surface. J. Fluid Mech. 197 (1988) 201-240. E57

[19] F. T. Smith and R. J. Bodonyi. Nonlinear critical layers and their development in streaming-flow stability. J. Fluid Mech. 118 (1982) 165-185. E61

[20] K. Stewartson. Marginally stable inviscid flow with critical-layers. IMA J. Appl. Math. 27 (1981) 133-175. E65

[21] M. D. Thomas. The nonlinear stability of flows over compliant walls. J. Fluid Mech. 239 (1992) 657-670. E57 
[22] K. S. Yeo. The Stability of Flow over Flexible Surfaces. PhD Thesis (University of Cambridge, 1986). E57

[23] K. S. Yeo. The stability of boundary layer flow over a single and multi-layer viscoelastic walls. J. Fluid Mech. 196 (1988) 369-408. E57

[24] K. S. Yeo. The three-dimensional stability of boundary-layer flow over compliant walls. J. Fluid Mech. 238 (1992) 537-577. E57, E58, E71, E77 